\title{
ACCIÓN Y CONSTRUCCIÓN LÓGICA
}

TOMÁS BARRERO

Departamento de Filosofía

Universidad de los Andes (Colombia)

ta.barrero32@uniandes.edu.co

RESUMEN: En este artículo discuto la semántica de Davidson para oraciones de acción a partir de su análisis de las preposiciones como predicados de sucesos individuales, y señalo ciertas dificultades lógico-lingüísticas conectadas con la pretensión interpretativa y la indiferencia referencial de dicha propuesta. Dejando de lado el presupuesto de la individualidad de los sucesos e inspirado en Evans y Grice, propongo una semántica interpretativa diferente para esos casos y argumento en favor de una tesis constructivista de los sucesos en el discurso de acción.

PALABRAS CLAVE: oraciones de acción, sucesos y preposiciones, forma lógica y semántica interpretativa

SUMMARY: By considering Davidson's analysis of prepositions as defining individual events predicates, I argue against his semantics for action sentences and stress some logico-linguistic puzzles concerning both the interpretive pretension and the referential indifference of this proposal. Inspired by Evans as well as by Grice, I advance another interpretive semantics for those cases which does not take as assumption the individual character of events and argue for a constructivist approach to events in action discourse.

KEY WORDS: action sentences, events and prepositions, logical form and interpretive semantics

Physics did not cease to be physics by being mathematized; similarly, logic did not cease to be metaphysics by being mathematized.

Oswaldo Chateubriand, Logical Forms I

En este escrito desarrollo un argumento tan completo como he podido para mostrar que hay problemas en la conexión entre oraciones de acción y oraciones cuantificadas sobre sucesos individuales tal como Davidson la concibe, y para sugerir una propuesta alternativa. El argumento, que se establece en torno al caso de las preposiciones con verbos de movimiento, se desarrolla en cuatro etapas. En la primera sección quiero reconstruir algunas oraciones de acción que incluyen preposiciones y verbos de movimiento en el marco de la teoría davidsoniana del significado. Resalto la necesidad de establecer una forma lógica conectada con el esquema $\mathbf{V}$ y dependiente de cons- 
tantes lógicas para captar el uso efectivo de los hablantes mediante lo que denominaré "paráfrasis agentivas y oracionales". Con respecto a esa forma lógica recalco, además, el requisito puramente teórico de la indiferencia de los axiomas referenciales para sucesos. En la segunda sección presento mi crítica semántica principal mostrando la inconsistencia de sostener la indiferencia referencial $y$ la necesidad de paráfrasis adecuadas para algunas oraciones con preposiciones. $\mathrm{Si}$ admitimos las dos, a partir del esquema $\mathbf{V}$ tenemos consecuencias lógicas que no son interpretables sin restricciones importantes (i.e., abandonando la indiferencia referencial); si abandonamos la indiferencia referencial, entonces la forma lógica propuesta resulta insuficiente para la comprensión de ciertas oraciones de acción que involucran movimientos. En la tercera sección, inspirado en Grice, exploro la posibilidad de una semántica interpretativa y trascendente como la de Evans para algunas oraciones con preposiciones y para las oraciones de acción en general. En esta alternativa el énfasis está puesto en la clasificación en términos de categorías semánticas y no en las constantes lógicas ni en las restricciones metalingüísticas impuestas por el esquema V. Comparo el caso de las preposiciones con el de otras categorías semánticas presentes en el discurso de acción (como adverbios atributivos y adverbios de acción) y concluyo que, desde el punto de vista semántico, podemos abandonar tranquilamente la tesis davidsoniana de los sucesos como individuos genuinos en favor de los sucesos como entidades complejas. En la última sección muestro una forma de acomodar los resultados de la tercera sección en el marco de una metafísica constructivista en la que la clasificación semántica de la acción nos capacita para introducir sucesos no como individuos, sino como construcciones lógicas.

\section{Un análisis lógico-semántico de los sucesos en las oraciones de acción}

La tesis de Davidson que quiero discutir afirma que las oraciones de acción requieren sucesos para ser verdaderas y que, por lo tanto, éstos son entidades teóricas admisibles en semántica (Davidson 1967a). Para entender dicha posición quisiera reparar en las razones que la motivaron en el contexto de la teoría davidsoniana del significado. Me parece que esas razones pueden dividirse en dos grandes tipos: preocupaciones externas con respecto a cómo la teoría puede hacerse cargo de ciertos fenómenos lingüísticos cotidianos y cuestiones internas acerca de cuál es la forma más económica o simple que 
debe asumir dicha teoría. Comienzo mi exposición con las cuestiones externas.

a) Usos efectivos, forma lógica y esquema $\boldsymbol{V}$

Davidson parte de señalar que la forma lógica proveniente de un análisis gramatical superficial de las oraciones de acción no nos permite hacerle frente a ejemplos cotidianos de habla. Por ejemplo, la forma lógica de una oración como ${ }^{1}$

(1) 「Bruto mató a César $\urcorner$

verdadera si y sólo si Bruto mató a César no $\mathrm{es}^{2}$

(2) $(\exists x)(\exists y)$ Mató a $(x y)$,

que resulta verdadera para al menos una interpretación $V_{\text {Bruto-César }}$ de $x$ e $y$, por la sencilla razón de que (2) no nos permite capturar dos rasgos importantes del discurso cotidiano. En primer lugar, (2) no nos capacita para entender ciertas correlaciones anafóricas bastante naturales a partir de (1), por ejemplo:

(3) $\ulcorner$ Bruto lo hizo a sangre fría, lo hizo con una daga, lo hizo durante los idus de marzo.?

¿A qué se refiere "lo" y cómo podríamos capturar esa referencia usando (2)? Seguramente el referente del pronombre está conectado con un elemento de discurso anterior y (2) no nos capacita para identificarlo. ${ }^{3}$ En segundo lugar, (2) no nos permite dar razón de inferencias cotidianas como

(4) Bruto mató a César con un cuchillo. Entonces Bruto mató a César.

${ }^{1}$ Utilizo los símbolos $\ulcorner\ldots\urcorner$ como una forma de referirme a expresiones u oraciones del lenguaje natural con el objeto de obtener su forma lógica. Distingo así entre este uso y las comillas, que utilizo en forma laxa para referirme a una expresión sin pretensiones con respecto a su forma lógica.

${ }^{2}$ No pretendo afirmar que ésta sea la forma lógica de (1), sólo quiero señalar las ventajas comparativas de la propuesta de Davidson con respecto a una forma lógica que, como (2), no introduce variables para sucesos. Agradezco a uno de los evaluadores anónimos esta observación.

${ }^{3}$ Para una detallada discusión del problema de la referencia pronominal en el discurso de acción, véase Grice 1986, pp. 9-10. 
Este modelo de inferencia parece ser una de las intuiciones lingüísticas más importantes que Davidson quiere reconstruir en su teoría y lo hace mediante un recurso metodológico muy sencillo. La forma lógica de (1) debe surgir de algunas consideraciones con respecto a sus condiciones de verdad, por lo que debe ser posible encontrar un posible esquema $\mathbf{V}$ consistente con el uso anafórico y las inferencias intuitivas. El esquema en el que Davidson parece estar pensando es el siguiente:

$\left(\mathbf{V}_{(1)}\right)\ulcorner$ Bruto mató a César $\urcorner$ es verdadera si y sólo si hay un matar de Bruto a César,

que podemos parafrasear en nuestro metalenguaje, el español en este caso, de la siguiente manera:

Hay un matar de Bruto a César si y sólo si hay un suceso que es un matar de Bruto a César.

Por lo tanto, la forma lógica de (1) es

(5) ( $\exists z)$ Mató a (Bruto, César, z).

(5) es verdadera en términos tarskianos si y sólo si hay al menos una valoración $V_{e}$ tal que $V_{e}$ (Mató a (Bruto, César, e)) $=\mathbf{V}$ y $V_{e}$ (Mató a (Bruto, César, e)) $=\mathbf{V}$ si y sólo si existe una tripleta ordenada conformada por las denotaciones de "Bruto", "César" y " $z$ " que pertenece a la extensión de "Mató a" de acuerdo con esa interpretación.

Esta propuesta parece tratar los casos de anáfora de acuerdo con una visión ortodoxa, usando variables ligadas que hacen referencia a un elemento del discurso inmediatamente anterior, es decir, al mismo suceso mencionado en (1). ${ }^{4}$ Además, (5) permite obtener como consecuencias lógicas requeridas las inferencias intuitivamente aceptables. Por ejemplo, la inferencia intuitiva (4) se transforma en un caso de distribución del cuantificador existencial con respecto a la conjunción:

$\left(4^{*}\right)(\exists z)[$ Mató a (Bruto, César, $z) \wedge$ Con $($ un cuchillo, $\left.z)\right]=(\exists z)$ (Mató a (Bruto, César, $z)){ }^{5}$

${ }^{4}$ En este punto me baso en la discusión de una interpretación cuantificacional de las expresiones descriptivas desarrollada por Neale (1990, caps. 5 y 6).

${ }^{5}$ Usaré el símbolo "=" para denotar la noción de consecuencia lógica con la que se buscar formalizar la idea de inferencia intuitiva. Cuando el contexto de uso no sea formal, como en (4), optaré por la expresión española indicadora de conclusión "entonces". Agradezco a uno de los evaluadores anónimos esta observación. 
Este tipo de inferencia, según Davidson (1967a), nos capacita para detectar el significado del verbo "matar" a partir de los lugares que admite el predicado Mató a. Pero lo hace con dos restricciones importantes e interconectadas. La primera es que el verbo implícito en la paráfrasis de $\left(4^{*}\right)$ debe ser hacer y no ser y que ese verbo requiere el reconocimiento de un agente, contrario a lo que sucede en las oraciones que no involucran verbos de acción. Al respecto dice Davidson: "De ' $x$ voló velozmente' podemos inferir que hay algo que $x$ hizo que se hizo velozmente. Aquí, sin embargo, 'velozmente' no se predica de $x$, sino de algo que $x$ hizo; en pocas palabras, de una acción" (1985a, p. 294; la traducción es mía).

La segunda es que, una vez se obtiene la forma lógica, la paráfrasis es fundamental porque nos permite comprender el significado de la oración original. Este ejercicio de interpretación intuitiva es el que garantiza que la traducción de la oración original es adecuada y que la teoría del significado es explicativa. Sabemos que la paráfrasis es adecuada cuando garantiza que las consecuencias lógicas de la formalización son interpretables como oraciones del lenguaje natural: ${ }^{6}$

La moraleja de estas breves observaciones puede sintetizarse así: para determinar la forma lógica de una expresión verbal, reduzca el número de argumentos del predicado verbal subyacente al menor número que produzca, con términos individuales apropiados, una oración completa. Pero no piense que tiene una oración completa hasta que haya descubierto suficiente estructura como para hacer válidas todas las inferencias que usted considere que se deben a la forma lógica. (Davidson 1985a, p. 295; la traducción es mía.)

En adelante denominaré al primer requisito necesidad de una paráfrasis agentiva y al segundo necesidad de una paráfrasis oracional para la forma lógica de las oraciones de acción. Así, pues, tal como las fórmulas de cualquier otra teoría científica, la forma lógica es el punto medio que vincula el fenómeno observable (el uso en un determinado contexto de oraciones como (1)) con una comprensión teórica que involucra objetos no observables (las condiciones de verdad que incluyen sucesos). La teoría se pone a prueba en la comprensión del uso de oraciones como (1) si es capaz de encontrar directamente para

${ }^{6}$ Agradezco a Édgar Andrade su insistencia en la posición contraria: que la semántica davidsoniana no requiere este tipo de restricciones. Sin embargo, los textos de Davidson apoyan la interpretación de que la paráfrasis admisible es un requisito semántico fundamental, so pena de pasar por alto los criterios de adecuación de una teoría del significado para un lenguaje natural. 
cada una de esas oraciones un ejemplo del esquema $\mathbf{V}$ que explique y prediga el comportamiento verbal de los hablantes que emiten esas oraciones; o bien, dado el caso en que no sea posible encontrarlo, si es capaz de traducir la oración original a una oración que sea un ejemplo de dicho esquema (Davidson 1967a, p. 182).

\section{b) La referencia como concepto teórico}

Con respecto a las características internas de la teoría, quisiera señalar dos principios conectados con esta estrategia metodológica. El primero (Davidson 1977, 1990) es la idea de que el concepto de referencia no tiene un papel protagónico en la obtención de las condiciones de verdad. Sucede más bien lo contrario: la referencia se entiende en términos del esquema $\mathbf{V}$, como un concepto teórico. La pregunta por el valor semántico de expresiones suboracionales debe plantearse en términos de cómo contribuyen esas expresiones a las condiciones bajo las cuales la oración es verdadera. ${ }^{7} \mathrm{El}$ segundo tiene que ver con la referencia como concepto teórico y puede resumirse en palabras del propio Davidson (1995) cuando presenta su ensayo "Realidad sin referencia":

Una teoría de la verdad es puesta a prueba por teoremas que estipulan las condiciones bajo las cuales las oraciones son verdaderas; estos teoremas no dicen nada acerca de la referencia. El Ensayo 15, "Realidad sin referencia", sostiene por consiguiente que la forma en que una teoría de la verdad mapee expresiones no oracionales con objetos es indiferente en tanto que las condiciones de verdad no se vean afectadas. A qué objeto se refiere una oración particular, o a qué objeto se refiere un término, o para qué objetos es verdadero un predicado, son preguntas que no tienen respuesta. (1995, p. 22; las cursivas son mías.)

No hay axiomas referenciales privilegiados; cualquier axioma referencial que nos permita obtener las condiciones de verdad adecuadas es admisible. En el caso de (1) debe haber un conjunto de axiomas referenciales cuyo único requisito teórico es garantizar la verdad de (5). De acuerdo con esta posición, los axiomas referenciales son formales y sólo se tocan indirectamente (a través de las condiciones de

${ }^{7} \mathrm{Y}$ esas condiciones no son denotativas para Davidson, como lo muestra su ataque a la correspondencia entre oraciones y hechos (Davidson 1969b). En lo que sigue me baso en la provechosa discusión de este ataque y de las ideas davidsonianas sobre la referencia como concepto teórico desarrollada por Neale (200la, cap. 2; 2001b). En la última nota discutiré brevemente esta posición. 
verdad para oraciones) con la experiencia (el uso efectivo de oraciones en las emisiones de los hablantes). ${ }^{8}$ Esos axiomas preservadores de verdad contienen toda la ontología con la que nos compromete la semántica del lenguaje natural, todas las entidades teóricas necesarias para hacer semántica. Los objetos físicos y los sucesos individuales están allí, como lo requieren las condiciones de verdad de (1) o (4), por ejemplo; pero los hechos o los mundos posibles no están allí. La conclusión parece clara: objetos físicos y sucesos son todas las entidades que necesitamos para que nuestras oraciones sean verdaderas (Davidson 1977, p. 233). A continuación quiero mostrar cómo entran en tensión estas condiciones internas de indiferencia referencial con las condiciones externas de paráfrasis admisible para el caso que me interesa, el de las oraciones de acción.

\section{Paráfrasis, satisfacción y preposiciones}

Hay varios problemas difíciles e interesantes para la propuesta de Davidson. ${ }^{9}$ En aras de la brevedad he escogido, para ponerla a prueba, el tratamiento de las frases preposicionales (Davidson 1967a, p. 150), es decir, la posibilidad semántica de aislar las preposiciones de los verbos. Tomemos: ${ }^{10}$

(6) 「Colón navegó hacia América ?,

que de acuerdo con Davidson debemos formalizar como

(7) $(\exists x)$ (Navegó (Colón, $x$ ) \& Hacia (América, $x)$ ),

y que permite obtener como consecuencias lógicas

(8) $(\exists x)$ (Navegó (Colón, $x)$ )

y

${ }^{8}$ Otra restricción importante de una teoría semántica adecuada para el lenguaje natural es la finitud de los axiomas no lógicos (Davidson 1970b, p. 74); es decir, los axiomas referenciales para sucesos deben ser finitos. La importancia de esta observación será clara en la discusión de la propuesta de Evans.

${ }^{9}$ Por ejemplo, voy a tratar sólo de pasada el caso de adverbios atributivos como "rápidamente" y los adverbios de acción como "torpemente" (Austin 1957), y no me ocuparé de la gran incógnita de cómo extender esta propuesta para tratar "acciones rituales" y "acciones lingüísticas" (Austin 1956, 1975). Reservo esos temas para un trabajo futuro.

${ }^{10}$ En éste y otros puntos me baso en el minucioso trabajo de Grice (1986). Debo agradecer a David Rey su valiosa discusión de los ejemplos de Davidson. Los errores que persisten son míos. 


\section{(9) $(\exists x)$ (Hacia (América, $x)$ ).}

En una semántica tarskiana esto quiere decir que cualquier interpretación del vocabulario no lógico que haga verdadera a (7), debe hacer verdaderas a (8) y (9). Pero ¿̨cuándo es verdadera (9)? Si utilizamos la técnica de la sección anterior $V((\exists x)$ (Hacia (América, $x))=\mathbf{V}$ si y sólo si existe una valoración $V_{e^{\prime}}$ de $x$ tal que $V_{e^{\prime}}$ (Hacia (América, $\left.e^{\prime}\right)$ ) $=\mathbf{V}$. ¿Cómo podemos definir esta secuencia de satisfacción? Sabemos que debe ser un par ordenado del que la denotación de "América" y de " $x$ " hagan parte y que pertenece a la extensión de "Hacia" de acuerdo con esa interpretación. Pero cuando se intenta establecer en qué condiciones un par ordenado en efecto pertenece a la denotación de "Hacia", es decir, cuáles son las condiciones de paráfrasis admisible para esta consecuencia lógica de (7), parece que quedamos atrapados en un dilema.

El primer cuerno de ese dilema reza así: supóngase que queremos mantener la tesis de que no hay preferencia referencial. No podemos dar por cierto que haya algún tipo de restricción sobre los sucesos que satisfacen el predicado "Hacia"; cualquier axioma será admisible siempre y cuando preserve el valor de verdad de las oraciones en las que el predicado aparece. Ya que los axiomas referenciales afirman que debe haber algo para que algunas oraciones sean verdaderas, la paráfrasis adecuada para oraciones de acción se establece en términos de verbos como haber o ser.

Sucede entonces algo curioso: en (7) tenemos una formalización que parece capturar nuestros usos de la oración (6). (9) es una consecuencia lógica de (6), pero no entendemos sus condiciones de verdad porque, en contrario de lo que supone la teoría de Davidson, no es fácil encontrar una paráfrasis admisible en español para esa fórmula. "Hay un suceso que es en dirección a América", "hay un suceso que sucede en dirección a América" y formas similares de expresión tienen una estructura extraña, diferente de la de las paráfrasis que incluyen el verbo de la oración original. (8), por ejemplo, se puede parafrasear como "hay un navegar de Colón" donde "hay" interpreta un verbo de la oración original ("navegó"), pero en (9) no tenemos simetría entre la paráfrasis y el verbo porque simplemente no hay verbo. Como en tantos otros casos en que no hay verbo, Davidson parece utilizar un verbo implícito como "ser", "haber". De hecho, ante esta dificultad (Davidson 1967a, p. 160), propone una paráfrasis que, adaptada a nuestro ejemplo, rezaría "Hubo un suceso que incluye movimiento hacia América" o "Hubo un suceso caracterizado por ser hacia América". Esta interpretación tiene dos problemas: el 
primero es que no está garantizada por la estructura lógica y, en consecuencia, da la impresión de ser arbitraria: ¿qué rasgos estructurales garantizan que sólo debamos considerar valores de $x$ que sean movimientos, como lo hace Davidson? ¿No estamos presuponiendo esa interpretación en vez de garantizarla por la estructura? Si es necesario tenerla en cuenta para una paráfrasis adecuada ¿no debería hacer parte de esa estructura de alguna manera, como lo afirma explícitamente Davidson en una de las referencias de la sección anterior?

El segundo es que, de acuerdo con sus propios principios interpretativos, el verbo implícito en la paráfrasis de (9) y en todo el discurso de acción es "hacer" y "hacer" tiene una estructura diferente de cualquiera de las otras paráfrasis mencionadas. Cuando damos una paráfrasis de "hacer" en términos de "ser", no todos los rasgos del uno se preservarán en el otro, v.gr., aquellos rasgos que involucran algún tipo de cambio o proceso. Por ejemplo, "hay un suceso en América" o "hay un suceso con César" no parecen generar problemas, porque la combinación del verbo "haber" con estas preposiciones y estos nombres propios es natural, mientras que "hay un suceso hacia América", "hay un suceso con cuchillo" parecen menos comprensibles que sus contrapartidas agentivas "se hizo hacia América" o "se hizo con un cuchillo", porque el verbo "haber" tiene un connotación estática de la que "hacer" carece y porque "hacer" es un verbo transitivo que admite naturalmente ciertos complementos circunstanciales, exóticos para "haber". Parece, entonces, que la tesis de la indiferencia referencial entra en conflicto con la tesis de la paráfrasis agentiva.

¿Cómo es posible, entonces, que pasemos en virtud de la forma lógica de una paráfrasis aceptable como "hay un suceso que es un navegar de Colón en dirección a América" a una paráfrasis extraña como "hay un suceso en dirección a América"? Mi hipótesis es que la propuesta de Davidson parece violentar una intuición sintáctica bastante sólida: que las preposiciones son expresiones sincategoremáticas. Eso explica que si bien entendemos (6), porque la conjunción garantiza la interpretación sincategoremática de la preposición "hacia", no podamos interpretar (9) muy naturalmente, porque hemos separado la preposición del verbo con el que parece formar una unidad. Si pensamos en el árbol sintáctico para (5), "hacia América" es una frase preposicional, es decir, una rama del sintagma verbal. En principio no tenemos evidencia directa en favor o en contra de que esa frase se pueda transformar en una oración completa, pero la dificultad de encontrar una paráfrasis para (9) nos inclina a pensar 
que una frase preposicional como ésta adquiere condiciones de oracionalidad cuando se adjunta al verbo que modifica. Así pues, la tesis de la indiferencia referencial entra en conflicto con la restricción de la paráfrasis agentiva porque la primera choca con la restricción de la paráfrasis oracional. La conclusión de esta primera parte del dilema es que (9) no puede por sí misma capturar un conjunto de condiciones de verdad porque las preposiciones no capturan por sí mismas condiciones de verdad; las capturan al unirse con alguna forma verbal, sea ésta personal o no. ${ }^{11}$

El segundo cuerno del dilema reza así. Supongamos que decidimos abandonar la tesis de la indiferencia referencial y asumimos la idea de que las condiciones de aplicabilidad para el predicado "Hacia" requieren una restricción a movimientos. En ese caso, parece claro que identificar un movimiento requiere identificar el objeto que se está moviendo, por ejemplo, identificar un movimiento de Colón. En su interesante discusión con Strawson sobre la prioridad de los objetos individuales en nuestro esquema conceptual, Davidson argumenta (1969a) en el sentido contrario, defendiendo un criterio causal para la identidad de los sucesos y señalando que la dependencia entre sucesos y objetos es de doble vía. Los sucesos requieren objetos porque los cambios requieren sustancias que cambien, pero los objetos requieren sucesos porque sólo a través del cambio es posible identificar una determinada sustancia. ${ }^{12}$ La tesis de Davidson se puede resumir así: si no hay prioridad conceptual entre sucesos y objetos, tampoco debe haber prioridad semántica alguna. Los axiomas referenciales para ob-

${ }^{11}$ Es necesario reconocer que, como señaló uno de los evaluadores del artículo, mi argumento en contra del tratamiento que Davidson les da a las preposiciones no es general. Se limita a un tipo de preposiciones que no encaja muy bien en la propuesta davidsoniana, preposiciones para las que la paráfrasis agentiva y la paráfrasis oracional parecen entrar en conflicto. Por el contrario, preposiciones que, como "en", tienen paráfrasis oracionales aceptables con verbos como "haber" son casos en favor del esquema davidsoniano aunque no tengan énfasis agentivo. Por ejemplo "hay un suceso en América" es una oración española impecable, aunque podamos discutir si especifica lo que alguien hizo.

${ }^{12}$ Hace falta precisar que Davidson abandona explícitamente el criterio causal para la identidad de los sucesos en un texto posterior (Davidson 1985b, p. 309), y luego de considerar la sugerencia de Quine de un criterio alternativo basado en la ubicación espacio-temporal, la descarta. Agradezco esta observación a uno de los evaluadores del artículo. Creo, sin embargo, que Davidson no ofrece una alternativa clara al criterio causal, a no ser que se entiendan sus observaciones del final de ese artículo referido como una invitación a encontrar un criterio de identidad alternativo "en nuestros predicados, nuestra gramática y nuestras formas de clasificar". El criterio alternativo que no encuentro en sus escritos y que estoy intentando discutir se basa en nuestros predicados, nuestra gramática y nuestra forma de clasificar. 
jetos siempre se pueden parafrasear en axiomas referenciales para sucesos. El paso de un lado al otro, sin embargo, es bastante discutible, porque depende de que exista una relación unívoca entre descripciones de un objeto en términos de los sucesos que lo afectan y descripciones de un suceso en términos de los objetos a los que afecta. En gracia de discusión, supongamos que podemos encontrar axiomas referenciales "paralelos" para los nombres en términos de los sucesos. De acuerdo con ellos, diríamos que en la secuencia de satisfacción para (9) el puede ser tomado como parámetro para fijar la referencia de "América". Que esa relación sea unívoca requiere encontrar implicaciones lógicas entre enunciados que hablan sobre sucesos y enunciados que hablan sobre objetos; según eso, en vez de (9) tendríamos la siguiente variante, lógicamente equivalente:

(9*) $(\exists x)(\mathbf{H a c i a}(x, e))$.

Pero no consigo imaginar una posible valoración $V_{a}$ restringida a sucesos que son movimientos tal que $V_{a}(\operatorname{Hacia}(a, e))=\mathbf{V}$. Esta descripción de la direccionalidad de los movimientos desde los movimientos mismos es extraña porque oscurece el sistema de coordenadas del que depende este rasgo de los movimientos. Creo que la evidencia lingüística, los usos efectivos de los hablantes, inclinan la balanza en favor de un sistema de coordenadas a partir del cual se asigna dirección a los movimientos. Ese sistema de coordenadas está dado en términos de los objetos que resultan conocidos para los hablantes y que se encuentran en su entorno. ${ }^{13}$ La localización de los movimientos como seres individuales sin recurrir a objetos individuales parece entonces una quimera, y la conclusión de que los objetos individuales son más primitivos que los sucesos — dados ciertos supuestos razonables - inevitable.

De este dilema concluyo que o bien es necesario modificar la forma lógica davidsoniana y probablemente discutir uno o varios de sus presupuestos metodológicos, o bien es necesario reconocer cierta asimetría semántica de los objetos con respecto a los sucesos. En la siguiente sección discuto el primer punto y en la última me ocupo del segundo.

${ }^{13}$ Deliberadamente he dejado de lado el problema - fundamental para Davidson - de la conexión entre forma lógica y aprendizaje por razones de extensión, pero creo que las dificultades para dar sentido de esa conexión con respecto a las preposiciones apoyan mi conclusión con respecto a la asimetría entre objetos individuales y sucesos. 


\section{Preposiciones y semántica interpretativa}

La pretensión de la teoría semántica de Davidson es explicar los usos efectivos de los hablantes a partir de una determinada estructura. Pero el caso de las preposiciones parece ponerla en duda al mostrar algunos inconvenientes en la comprensión de los usos efectivos a partir de la estructura asignada por la teoría. La sensación que se tiene es de arbitrariedad, porque la estructura parece estar orientada a garantizar ciertas consecuencias lógicas por incomprensibles -i.e., sin paráfrasis aceptables en español- que resulten. Evans (1976) ha diagnosticado agudamente el problema distinguiendo teorías semánticas trascendentes de teorías semánticas inmanentes, como la de Davidson. En esta sección quiero mostrar que la diferencia es útil en el caso de las preposiciones no sólo como diagnóstico, sino como programa de investigación.

Decimos que una teoría semántica es trascendente si, ante una expresión $e$ y un concepto teórico $W$, se pretende responder a la pregunta "¿Qué debería considerar una teoría como $W$ ?" a partir de lo que los hablantes que usan $e$ son capaces de comprender. Decimos que una teoría $\mathrm{T}$ es inmanente si estipula qué es $e$ de acuerdo con la teoría $W$. La forma de estipulación pasa por una definición recursiva o por una serie de definiciones recursivas que permiten construir todos los conceptos semánticos pertinentes con respecto a $e$ en el vocabulario de T. En el caso de Davidson y las oraciones de acción, tenemos el siguiente proceso. A partir de una definición recursiva (como la de Tarski) construimos cláusulas explícitas de satisfacción y verdad relativas a la estructura del metalenguaje elegido. Tomando como base un fragmento determinado y para el cual esas cláusulas están explícitas, abordamos las oraciones de acción con preposiciones. Dado que el fragmento está construido solamente para expresiones teóricamente reglamentadas — constantes lógicas y cuantificadores-, debemos traducir las oraciones de acción a una estructura con constantes lógicas y cuantificadores según la teoría - debemos encontrar una estructura relativa al metalenguaje- En el paso de (7) a (8) y (9), por ejemplo, necesitamos primero la traducción de (6) a un fragmento reglamentado (eso lo conseguimos con (7)) y, segundo, una prueba en el metalenguaje de la inferencia de (7) a (8) y (9). Fue en este punto donde se detuvo nuestro análisis ante el problema de encontrar paráfrasis adecuadas en español, o, en términos de Evans (1976, p. 70), una teoría homofónica de la verdad para el español. El resultado, recuérdese, es que \& es una representación semántica inadecuada para capturar las propiedades inferenciales de algunas 
preposiciones. La alternativa esquemática que sugerí fue delimitar los axiomas referenciales para las oraciones de acción acotando las interpretaciones posibles de las variables de suceso a la clase de los movimientos, aunque dicha restricción todavía es insuficiente.

Creo que esta alternativa puede compararse productivamente con las tesis de Evans de una semántica interpretativa (y trascendente) para el español por varias razones. En primer lugar, abandono la pretensión de una idea de la validez que se da en términos de la estructura de los verbos de acción (las inferencias del tipo (7) $\models$ (9)) usando constantes lógicas (como \&) y, en segundo lugar, lo hago señalando la necesidad de una clasificación semántica previa de expresiones como "hacia" para poder determinar de qué tipo son las inferencias en las que participan. En otras palabras, se requiere una clasificación de las preposiciones que nos permita determinar cómo las aplicamos, cuáles son sus propiedades semánticas esenciales y qué "temas" tienen en común (Evans 1976, p. 81). Esta clasificación debe tener en cuenta si la preposición se usa con un verbo de movimiento o de estado ${ }^{14}$ y qué inferencias se pueden validar a partir de esas interpretaciones admisibles. A la clasificación puramente formal de esas inferencias usando constantes lógicas deberíamos oponer una taxonomía que explicara las inferencias como parte esencial de la contribución de las preposiciones al significado de las oraciones de acción (Grice 1986, p. 23). En lo que resta de la sección quisiera discutir una explicación así y descartar tratamientos de las preposiciones poco promisorios.

\section{a) Preposiciones como predicados}

En lo fundamental ésta es la propuesta de Davidson y está afectada por los problemas que he señalado en la sección anterior. Si quisiéramos defender una alternativa atada a la letra davidsoniana de leer la estructura verbal como primitiva y los modificadores como adherencias separables de esa estructura, me parece que la opción más natural sería pensar en las preposiciones como expresiones generadoras de predicados sui generis y dependientes de la clase de interpretación, tal como los adjetivos atributivos ("alto"). El problema está en que

${ }^{14}$ Véase Grice 1986 (pp. 19-22) para una clasificación de este tipo con construcciones preposicionales de complejidad diversa. Si pensáramos esta propuesta en términos de la teoría semántica que se propone desde la lingüística (Katz 1979, cap. 3), diríamos que las implicaciones semánticas se explican en términos de las categorías semánticas (hormantes) y sus divisiones internas (restricciones seleccionales) y no a partir de un conjunto de expresiones lógicamente privilegiadas. 
Davidson reconoce no tener una propuesta satisfactoria para esos casos, por lo que Evans ha usado justamente "alto" como contraejemplo de una semántica inmanente. Si abandonamos la semántica inmanente y optamos por la semántica trascendente e interpretativa de Evans, difícilmente estamos en mejor posición porque no encontramos la manera de aplicar la técnica con la que tratamos adjetivos y adverbios atributivos. En efecto, si digo

(10) $\ulcorner$ Juan es alto $\urcorner$

$\mathrm{O}$

(11) $\ulcorner\text { Juan X-a/e rápidamente }\urcorner^{15}$

la semántica interpretativa de Evans propone condiciones acotadas de satisfacción. Para (10) tenemos la siguiente cláusula (Evans 1976 p. 68):

Para todos los predicados monádicos $\wp$ (posiblemente complejos), Juan satisface "alto"[ø] si y sólo si Juan es un satisfactor alto de $\wp$.

Mutatis mutandi, en el caso de (11) parece que podemos encontrar condiciones acotadas de satisfacción para sucesos y verbos de acción:

Para todos los predicados diádicos $\wp$ (posiblemente complejos), el X-ar/er/ir de Juan satisface "rápido"[ø] si y sólo si el Xar/er/ir de Juan es un satisfactor rápido de $\wp$.

La simetría entre (10) y (11) parece un punto en favor de tratar de la misma manera adjetivos atributivos y adverbios atributivos, pero no se aplica muy naturalmente a preposiciones. ${ }^{16} \mathrm{El}$ primer ajuste con respecto a Davidson es aumentar la aridad de los predicados

${ }^{15}$ Donde X-a/e es la representación formal de un verbo cualquiera en la tercera persona del singular del indicativo presente y X-ar/er/ir es su modo infinitivo.

${ }^{16}$ Como uno de los evaluadores del artículo me ha hecho notar, la propuesta de Evans con respecto a adjetivos y adverbios atributivos ha sido criticada con argumentos muy dignos de consideración por seguidores del programa de Davidson, como Lepore y Ludwig (2009, pp. 173-174). Su refutación se basa en los problemas que tiene la propuesta de Evans cuando dos adjetivos atributivos y "dependientes" ("topic dependent adjectives", como los llaman Lepore y Ludwig (2009, p. 162)) como "alto" y "gordo" se combinan en una misma oración. Las cláusulas de satisfacción que propone Evans no consiguen explicar la segunda cláusula de satisfacción sin recurrir a la interpretación de la primera; i.e., no podemos evaluar oraciones como "Colón es un hombre gordo alto" (en una lectura no conjuntiva) sin haber 
susceptibles de modificación para dar cabida al sintagma nominal (el movimiento es de alguien o algo); el segundo ajuste es una operación de concatenación sobre el predicado resultante para poder capturar la idea de dirección. Así, "hacia" hipotéticamente tendría las siguientes condiciones de satisfacción:

Para todos los predicados diádicos $\wp$ (posiblemente complejos), el X-ar/er/ir de Y satisface "haciaz"[ø] si y sólo si el X-ar/er/ir de $\mathrm{Y}$ es un satisfactor en dirección a $\mathrm{Z}$ de $\wp$.

La pregunta natural es cuál es el mecanismo de concatenación para este tipo especial de predicados. La restricción de interpretaciones de los adjetivos y adverbios atributivos no opera aquí, porque la relación de concatenación hacia ${ }_{z}$ no es interna; i.e., no determina un subjconjunto relevante de interpretaciones para las cuales se satisface el predicado. La relación preposicional parece de tipo funcional, como la generada por un operador con un parámetro fijo (la coordenada espacial en este caso) que toma oraciones como argumentos y genera oraciones como resultado. ¿Pueden leerse entonces las preposiciones como funciones oracionales en papel adverbial? Creo que no, al menos si tomamos el modelo más reconocido de las funciones oracionales en el discurso de acción, como mostraré a continuación.

\section{b) Preposiciones y funciones oracionales}

Las funciones oracionales conectadas con modificación adverbial están asociadas con lo que Davidson denomina "adverbios de acción". ${ }^{17}$ ¿Podríamos entonces extender la propuesta de Davidson para adverbios de acción ("intencionalmente", "voluntariamente") para el caso de las preposiciones? A diferencia de los predicados extensionales usuales, los adverbios de acción presentan la acción desde una perspectiva, en una descripción posible. Por ejemplo:

\section{(12) $\ulcorner$ Colón navegó intencionalmente hacia América $\urcorner$}

interpretado "Colón es un hombre gordo" y eso implica la introducción de una infinidad de axiomas de satisfacción para cada uno de los adjetivos atributivos y sus posibles combinaciones. Una teoría de la verdad para el español con infinitos axiomas parece un esperpento teórico. Sin embargo, mi interpretación de Evans para el caso de las preposiciones no requiere que yo tenga por válido su análisis de los adjetivos y adverbios dependientes, sino su aparato de funciones. La importancia de esta restricción se verá más adelante.

${ }^{17}$ Esta propuesta no se encuentra en Davidson y la estoy usando con pretensiones puramente comparativas e ilustrativas, como un caso más de modificación adverbial. 
tiene la forma lógica (Davidson 1967a, p. 154).

(13) Fue intencional por parte de Colón que $(\exists x)$ (Navegó (Colón, $x)$ \& Hacia (América, $x$ )).

Pero hay un problema para ver las preposiciones como funciones oracionales de este tipo: uno puede hablar de una acción en su descripción intencional, pero decir que un movimiento es dirigido equivale a dar una clasificación de ese movimiento no sujeta a creencias o actitudes del agente. Podemos discutir sobre sistemas diferentes de coordenadas, pero esos sistemas no son relativos a las creencias de alguien en el sentido en que (13) es relativo a las creencias y actitudes de Colón, porque su punto de vista con respecto a navegar hacia América es central para determinar el valor de verdad de (13), en tanto que no tiene importancia alguna para determinar el valor de verdad de (7). La perspectiva que necesitamos para entender las preposiciones no es la del agente qua agente, sino la del agente qua objeto material en un sistema de coordenadas.

\section{c) Preposiciones y funciones}

Necesitamos encontrar asignaciones apropiadas para interpretar verbos de movimiento. Esos verbos pueden verse como una categoría semántica que toma sus valores en conjuntos de objetos, tiempos y lugares y, por ende, no es exclusiva del discurso de acción. En esa categoría están todos los ingredientes para capturar la idea de direccionalidad. "Hacia" se definirá entonces como una función que va de conjuntos de objetos materiales, tiempos y lugares en conjuntos de objetos materiales, tiempos y lugares. ${ }^{18}$ Llamemos $I_{\text {mov }}$ esa interpretación y restrinjamos su dominio a $I_{m o v}^{\prime}$, el conjunto de objetos animados, tiempos y lugares. Por ejemplo:

(14) $\ulcorner$ Juan X-a/e hacia Z $\urcorner$,

tiene las condiciones de satisfacción:

Para todos los conjuntos $<\mathrm{Juan}, t_{1}, l_{1}>$ y $<$ Juan, $t_{1+n}, l_{2}>$, el $\mathrm{X}$-ar/er/ir de Juan en $t_{1}$ y $l_{1}$ y el X-ar/er/ir de Juan en $t_{1+n}, l_{2}$ satisfacen "hacia Z" si existe una función $f$ tal que el X-ar/er/ir de Juan en $t_{1+n}, l_{2}$ es el satisfactor de $f$ en dirección a $Z$ para $<$ Juan, $t_{1}, l_{1}>$.

\footnotetext{
${ }^{18}$ Esta restricción temporal es fundamental si queremos conservar la idea intuitiva de que los movimientos son cambios de los objetos.
} 
Si se acepta esta definición, podemos estipular la función inversa $f^{-1}$ que permite definir la interpretación para "desde". Dado que ninguna interpretación de "hacia" coincide con alguna interpretación de "desde" para los mismos argumentos, toda interpretación que haga verdadera a (14) hará falsa a (15): ${ }^{19}$

(15) $\ulcorner$ Juan X-a/e desde $Z$ ?

Podemos explicar entonces la validez de toda una gama de inferencias intuitivas y puramente preposicionales (con las restricciones temporales y espaciales obvias) como la siguiente:

(16) $\ulcorner$ Juan $X$-a/e/o hacia $Z\urcorner$ entonces $\ulcorner$ Juan no $X-a / e / o$ desde $Z\urcorner$.

En una formulación menos críptica, podemos explicar la validez de inferencias estructurales a partir de (6) como ésta (las restricciones temporales y espaciales obvias):

(17) 「Colón navegó hacia América $\urcorner$ entonces $\ulcorner$ Colón no navegó desde América $\urcorner$.

Creo que ajustando ligeramente la definición podríamos incluir la mayoría de preposiciones españolas en su sentido espacial y/o temporal más básico, aunque no tengo claro todavía cómo tratarlas en sus usos metafóricos. El tema que las preposiciones "hacia" y "desde" tienen en común es el de la dirección y son las atribuciones de dirección lo que aprendemos al usar dichas expresiones. La semántica interpretativa que sugiero no requiere sucesos individuales para explicar las preposiciones ya que toma tripletas ordenadas de objetos físicos, tiempos y lugares como argumentos, y plantea así un desafío a otra tesis de Davidson, la de considerar las propiedades temporales como propiedades de sucesos individuales (Davidson 1967b, pp. 200-201). Podemos hacer un análisis semántico de las oraciones de acción en particular, y de las oraciones de movimiento en general, sin recurrir a una categoría de sucesos individuales y partiendo directamente de las categorías semánticas a las que pertenecen los verbos. En nuestra explicación, las constantes lógicas no son privilegiadas ni centrales: las inferencias (16) y (17) no dependen de las propiedades de la negación, sino de la incompatibilidad de las asignaciones estipuladas para las funciones $f$ y $f^{-1}$. En ese sentido, nuestra propuesta es

${ }^{19}$ Aunque uno de los evaluadores de Crítica sugiere que un movimiento puede ser en el mismo instante y en la misma ubicación espacial desde y hacia América, confieso que no veo cómo podría suceder esto, dadas las restricciones mencionadas. 
trascendente: no depende de las características particulares de la notación semántica canónica (lógica de primer orden con identidad) ni queda presa de ella. Además, le da sustento a la intuición de que las preposiciones son expresiones sincategoremáticas pero, a diferencia de otras expresiones sincategoremáticas como las constantes lógicas, la complejidad preposicional es dependiente del tipo de verbo al que modifican. No creo que esos tipos generen los mismos problemas de la propuesta de Evans con respecto a los adjetivos atributivos, porque no creo que cada verbo genere una relación de satisfacción particular con cada preposición dado que (i) los tipos de verbos son finitos, (ii) el número de proposiciones es finito, y (iii) las condiciones de satisfacción para preposiciones sólo dependen de (i) y (ii). ${ }^{20}$ En la siguiente sección explotaré estos resultados semánticos para defender una cierta diversidad ontológica entre clases de sucesos.

\section{Los sucesos como construcciones lógicas}

No cabría decir con propiedad que el discurso de acción no contiene una referencia velada a sucesos. Pero, como Austin ya lo mostró suficientemente en el caso de los modificadores adverbiales (Austin 1957), el énfasis no debe estar en "suceso", sino en "referencia velada". Cuando se examina el caso de las preposiciones, se tiene la impresión de que los sucesos tienen una función diferente de la de términos referenciales genuinos; parecen constructos dependientes de otros objetos (como los objetos físicos, los tiempos y los instantes). ¿De dónde proviene el que esas funciones se hayan confundido en la propuesta de Davidson? De una estrategia metodológica sospechosa: sustituir entidades teóricas familiares por entidades inferidas. Hay un pasaje de la presentación del atomismo lógico donde Russell propone un criterio heurístico de admisibilidad para entidades basado en la idea de construcción lógica y que reza así: "Siempre que sea posible, sustitúyase construcciones surgidas de entidades conocidas por inferencias a entidades desconocidas" (Russell 1924, p. 40).

Russell sostiene que se trata de una versión de la navaja de Occam y que su aplicación ha sido exitosa en el campo de la lógica matemática y del análisis lógico del lenguaje. Si podemos preservar la verdad

${ }^{20}$ Por limitaciones de espacio sólo he desarrollado mi propuesta para un caso particular. Con un poco de atención al detalle, ingenio y tiempo, creo que la propuesta se puede extender utilizando las herramientas suministradas por Evans y siguiendo las intuiciones de Grice con respecto a otras preposiciones (Grice 1986, pp. 20-21). Probablemente esta extensión implique encontrar, para cada preposición, un tipo de función que capture sus condiciones de satisfacción. 
en el paso de construcciones a inferencias, en el fondo las entidades inferidas no son necesarias porque no están presupuestas lógicamente en esa clase de discurso. El caso interesante se da cuando no podemos preservar la verdad. Entonces parece haber una estrategia metodológicamente cuestionable y cierta área de nuestro discurso se poblará de entidades sospechosas. El uso de sucesos en oraciones de acción que no involucran preposiciones es aparentemente ${ }^{21}$ un caso de entidades conocidas (sucesos individuales), pero - he argumentado - la inferencia de esas entidades individuales a sucesos individuales en oraciones con preposiciones no se sostiene, dada la evidencia lingüística.

Este paso indebido puede entenderse más como un compromiso teórico irrestricto con los sucesos como individuos que como una necesidad impuesta por el análisis semántico. Podría de hecho haber otro tipo de razones (no semánticas) para sostener la uniformidad de los sucesos individuales a través de todo el discurso de acción, razones a las que me referiré al final de este trabajo. En el campo estrictamente semántico, si hubiera una forma menos ad hoc de conectar nuestro discurso sobre acciones con nuestro discurso sobre sucesos en términos de un concepto o grupo de conceptos que señalara la asimetría entre entidades familiares y entidades construidas, la sensación de arbitrariedad disminuiría. Creo que de hecho existe tal concepto y que Grice lo ha señalado con claridad:

Mi intuición lingüística me dice que si te digo que tengo 93, yo (en condiciones normales) te estoy diciendo mi edad (diciéndote cuántos años tengo), y no hablando sobre mi edad; y, en analogía, que si te digo que dos automóviles chocaron en el puente, yo (en condiciones normales) te estoy diciendo (estoy contándote) lo que sucedió, no describiendo o hablando sobre o diciendo algo acerca de lo que sucedió. De hecho, decir lo que sucedió parece no sólo ser distinto de hablar sobre lo que sucedió, sino, en cierto sentido, estar presupuesto por ello; decir lo que sucedió parece ser, de un modo intuitivo, especialmente central; es algo que necesitamos hacer para informarle a un oyente de qué estamos hablando cuando hablamos de lo que sucedió. [...] En el análisis de Davidson, pienso, no hay lugar para la distinción que estoy defendiendo; desde su punto de vista, todo lo que uno dice de lo que sucedió es (en efecto) un caso de decir que algo sucedió que - . (Grice 1986, p. 15; la traducción es mía.)

${ }^{21}$ Aparentemente porque creo que adverbios como "torpemente" también pueden generarle problemas a la propuesta de Davidson, pero no tengo espacio para presentarlos o discutir el asunto en este escrito. 
Nuestro discurso de acción es central en el sentido de que son las incompatibilidades interpretativas entre, por ejemplo, verbos y preposiciones las que determinan la naturaleza de los sucesos que aparecen en la forma lógica. Si mi argumento con respecto a las preposiciones es correcto, me parece razonable afirmar que las categorías semánticas están presupuestas en nuestra descripción de lo que sucede y concluir de ahí que los sucesos son construcciones lógicas a partir de una clasificación previa del discurso de movimiento y del discurso de acción. El lenguaje de acción es aquel en el que usamos los verbos de acción con toda su carga semántica habitual; el lenguaje de suceso es aquel en el que hablamos sobre o mencionamos el discurso de acción con el objetivo de estipular algunas propiedades a partir de las cuales identificamos lo que sucede. En la parte de ese mismo pasaje que no he citado, Grice sugiere que la dificultad de Davidson para apreciar esta diferencia de niveles proviene de sus dificultades para valorar en todo su detalle la propuesta de Reichenbach con respecto a sucesos. Creo que ese diagnóstico de Grice es correcto y que nos da una pista importante no sólo sobre la conexión entre el discurso de acción y el discurso sobre sucesos, sino sobre algunas razones que llevan a Davidson a identificarlos.

Reichenbach (1952) sostiene que la idea de individuo es relativa al contexto teórico en el que trabajamos. Cuando las necesidades de nuestro discurso así lo ameritan, estipulamos una convención para una nueva clase de individuos. En nuestro discurso cotidiano, por ejemplo, parecemos comprometidos con clases del tipo cosa, como señala Davidson correctamente, pero en el discurso de la física moderna nos impulsa a suponer que hay individuos del tipo suceso. Los primeros son "agregados de materia que se mantienen juntos durante cierto tiempo"; los segundos son "coincidencias de espacio-tiempo y no permanecen" (Reichenbach 1952, p. 267); los primeros son primitivos en nuestro discurso cotidiano, mientras que los segundos son primitivos en nuestro discurso físico. Lo que necesitamos es una manera de conectar estas dos formas de hablar sobre el mundo, y Reichenbach nos propone esa estrategia que creo que se puede interpretar coherentemente en términos de la propuesta de una semántica interpretativa para el español. ${ }^{22}$ En esa semántica tenemos una cate-

${ }^{22}$ Digo "se puede interpretar" porque la semántica de Reichenbach descansa en el tipo de conceptos tradicionales ("forma lógica", "equivalencia lógica", etc.) que la semántica interpretativa considera secundarios e inmanentes. Lo que sigue puede verse como la fundamentación interpretativa y trascendente de las equivalencias semánticas detectadas por Reichenbach en un nivel puramente formal. Deliberadamente he dejado de lado un punto importante en esa propuesta: la idea de que hay 
goría de movimientos; algunos de ellos tienen que ver con acciones, muchos otros no. (6) es un ejemplo de los primeros, mientras que (18) es un ejemplo de los segundos:

(18) $\ulcorner$ El viento sopló hacia América $\urcorner$.

(6), según Reichenbach, es lógicamente equivalente a (19), mientras que (18) es equivalente a (20):

(19) "La navegación de Colón tuvo lugar hacia América",

(20) "El viento se movió en dirección a América".

Ahora bien, si los sucesos son coincidencias instantáneas de espacio y tiempo, (18), (19) y (20) contienen una referencia explícita a sucesos, pero (6), nuestra única oración de acción, no. Las oraciones de acciones que involucran movimientos se distinguen justamente por esta posibilidad de eliminar los sucesos que entonces dejan de ser individuos genuinos en el discurso de acción y pasan a ser construcciones lógicas. Si volvemos a nuestra propuesta de condiciones de satisfacción para oraciones del tipo (14) (como (6)), deberemos notar una diferencia sutil con oraciones como (18): el primer término de la tripleta ordenada en el primer caso es del tipo "objeto individual", mientras que en el segundo es una tupla ordenada que delimita, en este ejemplo, la región espacial del aire que llamamos "viento". La clase de movimientos en cuyo análisis no son eliminables los sucesos coincide con la clase de movimientos donde la primera coordenada no es un objeto físico, sino una tupla ordenada de coordenadas espaciales y/o espacio-temporales. Tal vez este resultado sugiera que la primera clase donde los sucesos sí son eliminables coincide con las tripletas ordenadas en las que el primer término es un objeto animado. Los

un operador lógico, "es un hecho que", que nos permite pasar de oraciones tipocosa (6) a oraciones tipo-suceso (19) (Reichenbach 1952, p. 269). Son ampliamente conocidas las críticas de Davidson a las semánticas que recurren a hechos o situaciones. Esas reservas se formulan varias veces en su obra mediante la construcción de un argumento tipo slingshot (Davidson 1967a, 1969b) que, por ejemplo, Grice critica sugiriendo una restricción para operadores lógicos que mencionan hechos (Grice 1986, pp. 5-6). Aunque mi discusión no se ve afectada directamente por esa disputa, creo que esa sugerencia es todo lo que se necesita para los argumentos slingshot. Como Neale (200la) lo ha mostrado suficientemente, podemos admitir estados de cosas en semántica sin peligro de caer presa de argumentos de este tipo si somos capaces de encontrar las restricciones adecuadas con respecto a la sustitución salva veritate y a la sustitución por equivalentes deductivos, y las restricciones son condiciones constitutivas de cualquier regla de inferencia. No hay nada especialmente preocupante con los hechos como entidades semánticas. 
movimientos así delimitados son las acciones de seres que tienen una capacidad orgánica de moverse. Una discusión interesante es qué papel desempeñan las condiciones de identidad de ese objeto animado en una propuesta de los sucesos como estructuras lógicas y no como individuos (Montague 1969). En otro trabajo he argumentado que también desde las condiciones de identidad, la asimetría entre objetos individuales y constructos lógicos se preserva en el discurso de acción (Barrero 2011).

Existe, sin embargo, la posibilidad de que debamos interpretar las tesis de Davidson sobre la simplicidad de los sucesos no en términos de cuán adecuadamente se maneja con ellos el habla cotidiana, sino en términos de cuán útiles resultan como evidencia para su análisis de enunciados causales que apoyen su teoría causal de la acción. Su discusión sobre la identidad de los sucesos (Davidson 1969a, 1985b) permite, en mi opinión, esa interpretación, aunque he señalado solamente de pasada esas razones en la segunda sección. Si ése es el orden correcto de lectura (de una concepción causal de la acción a una concepción semántica sobre las oraciones de acción), entonces creo que mi escrito puede verse como un alegato en favor de una concepción de la acción en la que la causalidad entre sucesos individuales no es la única clave teórica. Mi alegato sigue así en sus aspectos fundamentales las potentes intuiciones del de Austin en favor de las excusas, en cuanto a mostrar la clasificación causal de la acción como una entre varias posibles y no necesariamente como la más primitiva (Austin 1957). ${ }^{23}$

\section{BIBLIOGRAFÍA}

Austin J.L., 1979, Philosophical Papers, ed. J.O. Urmson y G.J. Warnock, Oxford University Press, Oxford.

— 1975, How to Do Things with Words, ed. J.O. Urmson y M. Sbisà, Harvard University Press, Cambridge, Mass.

— 1957, “A Plea for Excuses”, en Austin 1979, pp. 175-204.

—_, 1956, "Performative Utterances", en Austin 1979, pp. 233-252.

Barrero, T., 2012, "Acción, hecho y suceso", Manuscrito, vol. 35, no. 2, pp. 207-231.

${ }^{23}$ Este trabajo forma parte del proyecto de investigación "Lenguaje y acción. Fundamentos de una semántica multidimensional" financiado por la Universidad de los Andes. En una primera versión recibió valiosos comentarios de los miembros del grupo de investigación "Lógica, Epistemología y Filosofía de la Ciencia" de las universidades de los Andes y el Rosario, a quienes quiero manifestar mi profundo agradecimiento. 
Barrero, T., 2011, “Acción, identidad y sucesos", aparecerá en C. Patarroyo (comp.), Autoconocimiento: perspectivas filosóficas contemporáneas, Universidad del Rosario, Bogotá, 2013.

Chateaubriand, O., Logical Forms. Part I - Truth and Description, CLEUNICAMP, Coleção CLE, vol. 34, Campinas, 2001.

Davidson, D., 2002, Essays on Actions and Events, Clarendon Press, Oxford.

—_, 1995, Ensayos sobre acciones y sucesos, trad. O. Hansberg, J.A. Robles y M. Valdés, Crítica/Instituto de Investigaciones FilosóficasUNAM, Barcelona.

—_, 1990a, De la verdad y la interpretación, trad. Guido Filippi, Gedisa, Barcelona.

- $1990 \mathrm{~b}$, "The Structure and Content of Truth", The Journal of Philosophy, vol. 87, no. 6, pp. 279-328.

—_, 1985a, “Adverbs of Action”, en Davidson 2002, pp. 293-304.

311 .

1985b, "Reply to Quine on Events", en Davidson 2002, pp. 305-

—_, 1977, “Realidad sin referencia”, en Davidson 1995, pp. 219-228.

239.

—_, 1970b, "Semántica para lenguajes naturales", en Davidson 1990a, pp. 73-81.

—. 1969a, "La individuación de los sucesos", en Davidson 1995, pp. 206-207.

——, 1969b, "Fiel a los hechos", en Davidson 1990a, pp. 57-72.

—_, 1967a, "La forma lógica de las oraciones de acción”, en Davidson 1995, pp. 133-187.

—_, 1967b, "Relaciones causales", en Davidson 1995, pp. 189-206.

Evans, G., 1976, "Estructura semántica y forma lógica”, Ensayos filosóficos, trad. Alejandro Tomasini Bassols, Instituto de Investigaciones FilosóficasUNAM, México, 1996, pp. 63-90.

Grice H.P., 1986, "Actions and Events", Pacific Philosophical Quarterly, vol. 67, no. 1, pp. 1-35.

Katz, J., 1979, Teoría semántica, trad. J. García Puente, Aguilar, Madrid.

Lepore, E. y K. Ludwig, 2009, Donald Davidson's Truth-Theoretic Semantics, Clarendon Press, Oxford.

Montague, R., 1969, "Sobre la naturaleza de ciertas entidades filosóficas", Ensayos de filosofía formal, ed. R.H. Thomason, trad. J.D. Quesada, Alianza, Madrid, 1977, pp. 118-157.

Neale, S., 200la, Facing Facts, Oxford University Press, Oxford.

— , 2001b, "Meaning, Truth, Ontology", en P. Kotatko, P. Pagin y Segal Gabriel (comps.), Interpreting Davidson, CSLI, Stanford, pp. 155198.

, 1990, Descriptions, The MIT Press, Cambridge, Mass. 
Reichenbach, H., 1952, Elements of Symbolic Logic, Macmillan, Nueva York.

Russell, B., 1924, “Atomismo lógico", en A.J. Ayer (comp.), 1978, El positivismo lógico, trad. L. Aldana, U. Frisch, C.N. Molina, F.M. Torner y R. Ruiz Harrel, Fondo de Cultura Económica, México, pp. 37-56.

Recibido el 5 de octubre de 2011; revisado el 12 de junio de 2012; aceptado el 26 de julio de 2012. 